\title{
Research of Model Matching Control of Torque Vectoring Differential Gear System
}

\author{
Yutaka Hirano ${ }^{1}$ \\ ${ }^{1}$ Toyota Motor Corporation, Japan, yutaka_hirano@mail.toyota.co.jp
}

\begin{abstract}
In this paper, model-based development of a control of torque vectoring differential (TVD) gear system is described. A new control logic was developed using model matching control to let the vehicle yaw rate and vehicle slip angle follow the desired dynamics. Simulation results using a single track model of vehicle dynamics are shown to prove the efficacy of the proposed control. Modelica was useful to express timevarying state space system such as the single track model of vehicle dynamics. Also full vehicle model considering all of the vehicle dynamics and drive train motion using Modelica clarified the characteristics of this method in actual driving cases.
\end{abstract}

Keywords: Model Based System Development, Vehicle Dynamics, Torque Vectoring, Model Matching Control

\section{Introduction}

To satisfy needs for future low-carbon mobility society, development of many new electric vehicles (EVs) is increasingly active in recent years. Additionally many new proposals about integrated electric power train which also has torque vectoring capability are presented. Authors had made an integrated model of the total vehicle system of such an EV using Modelica (Hirano, 2014) (Hirano, 2015).

In the paper (Hirano, 2014), the authors showed the capability of new construction of the new EV using new type of tire based on 'Large and Narrow concept' and torque vectoring differential (TVD) gear. For the model based development of the new EV, various kind of running resistance, vehicle dynamic performance and proper design of electric regeneration system were studied. In another previous research (Hirano, 2015), a multi-physics full vehicle model of the new EV is expanded to consider the detailed loss of motors and inverters. Also front and rear suspension model which has same 3D mechanical design as the real experimental vehicle was made and verified. By technical investigations using this full vehicle model, structure, specifications and control of the new EV system were researched about vehicle dynamics and energy consumption. However, the control logic of the TVD gear was only simple PI feedback control in the previous papers. In this paper, model based control of TVD gear system is developed using model matching control technique. Single track model of vehicle dynamics is used to derive and verify the new control. At the same time, detailed design parameter of vehicle dynamics was obtained from the analysis of Modelica full vehicle model using detailed suspension model. Finally the developed controls were verified by using both the single track model and the full vehicle model.

\section{Specification of Experimental EV}

Table 1. Specifications of new experimental EV

\begin{tabular}{|c|c|c|}
\hline & New EV & $\begin{array}{c}\text { Conventional } \\
\text { car }\end{array}$ \\
\hline Vehicle Weight & $750 \mathrm{~kg}$ & $1240 \mathrm{~kg}$ \\
\hline Yaw Moment Inertia & $869 \mathrm{kgm}^{2}$ & $2104 \mathrm{kgm}^{2}$ \\
\hline Wheelbase & $2.6 \mathrm{~m}$ & $2.6 \mathrm{~m}$ \\
\hline $\begin{array}{c}\text { Front : Rear Weight } \\
\text { Distribution }\end{array}$ & $0.48: 0.52$ & $0.62: 0.38$ \\
\hline Height of CG & $0.38 \mathrm{~m}$ & $0.55 \mathrm{~m}$ \\
\hline $\begin{array}{c}\text { Aerodynamic Drag } \\
\times \text { Frontal Area }\end{array}$ & $0.392 \mathrm{~m}^{2}$ & $0.644 \mathrm{~m}^{2}$ \\
\hline Tire RRC & $5 \times 10^{-3}$ & $8.8 \times 10^{-3}$ \\
\hline Tire Normalized CP & 16.1 & 20.4 \\
\hline
\end{tabular}

The proposed experimental EV has specifications as shown in Table 1 (Hirano, 2015). Compared with a conventional small-class passenger car, the new EV has characteristics of lighter vehicle weight, smaller yaw moment of inertia, lower height of the center of gravity (CG) and lower rolling resistance coefficients (RRC) of tires. Because of these characteristics, this new EV is expected to have better handling and lower energy consumption than conventional vehicles. On the other hand, because of lighter weight and lower value of tire normalized CP (Cornering Power), this new EV seems more sensitive against external disturbances such as crosswind and road irregularity than the conventional cars. To cope with this problem, direct yaw moment control (DYC) was applied by using a new integrated transaxle unit for rear axle which has a main electric motor and also TVD gear unit with a control motor. 


\section{Vehicle Model for Controller Design}

\subsection{Single Track Vehicle Model}

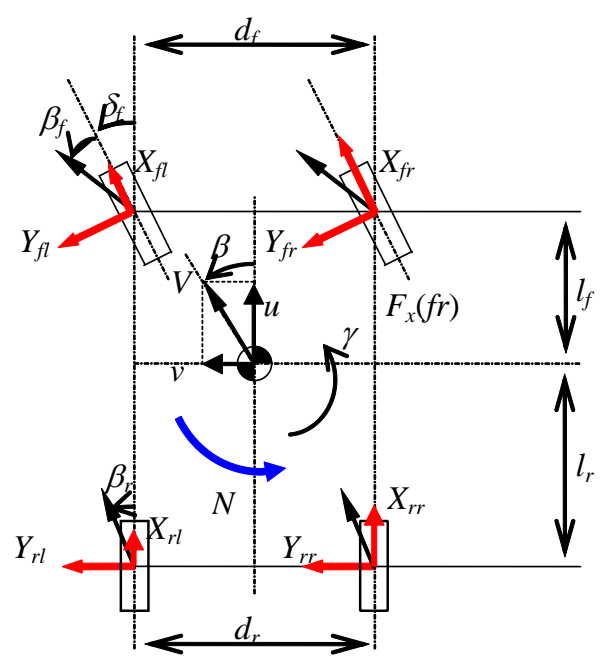

Figure 1. Expanded single track vehicle model

Figure 1 shows an expanded single track vehicle dynamics model to derive the control logic. The simplified equations of motion by this model become as follows.

$$
\begin{aligned}
& M \frac{d V}{d t}=F \approx\left(X_{f r}+X_{f l}\right) \cos \delta_{f}+\left(X_{r r}+X_{r l}\right) \\
& M \frac{d}{d t}\left(V \tan ^{-1} \beta+V \gamma\right) \approx Y_{f l}+Y_{f r}+Y_{r l}+Y_{r r} \\
& I_{z} \frac{d \gamma}{d t} \approx l_{f}\left(Y_{f l}+Y_{f r}\right) \cos \delta_{f}-l_{r}\left(Y_{r l}+Y_{r r}\right)+N \\
& N=d_{f}\left(X_{f r}-X_{f l}\right) \cos \delta_{f}+d_{r}\left(X_{r r}-X_{r l}\right)
\end{aligned}
$$

Here,

$$
\begin{array}{ll}
\beta & : \text { Vehicle slip angle, } \\
\gamma & \text { : Vehicle yaw rate, } \\
M & \text { : Vehicle mass, } \\
V & \text { : Vehicle velocity, } \\
I_{z} & \text { : Vehicle yaw moment of inertia, }
\end{array}
$$

$l_{f}\left(l_{r}\right)$ : Distance from the CG to front (rear) axle, (CG: Center of Gravity)

$d_{f}\left(d_{r}\right)$ : Tread of front (rear) axle,

$X_{* * *} \quad$ : Longitudinal force of each tire,

$Y_{* *} \quad:$ Lateral force of each tire,

$\delta_{f} \quad$ : Steering angle of front tire,

$F \quad$ : Vehicle driving force,

$N \quad$ : DYC moment by TVD.

\subsection{Equation of Motion for Vehicle Dynamics}

To derive the equations of motion for the target vehicle, equations (1) to (4) were further simplified. The lateral force at left and right tires were assumed to be equal and let $Y_{f l}=Y_{f r}=Y_{f}, \quad Y_{r l}=Y_{r r}=Y_{r}$. Also we assume $\cos \delta_{f} \approx 1$ when front tire steering angle is not so big, and $\tan ^{-1} \beta \approx \beta$ when $\beta$ is small. Also by considering the TVD power unit is equipped only in the rear axle, the equations of motion become as follows.

$$
\begin{aligned}
& M \frac{d V}{d t}=F=\left(X_{r r}+X_{r l}\right) \\
& M V\left(\frac{d \beta}{d t}+\gamma\right)=2 Y_{f}+2 Y_{r} \\
& I_{z} \frac{d \gamma}{d t}=2 l_{f} Y_{f}-2 l_{r} Y_{r}+N
\end{aligned}
$$

where

$$
\begin{aligned}
& Y_{f}=-K_{f} \beta_{f}=-K_{f}\left(\beta+\frac{l_{f}}{V} \gamma-\delta_{f}\right) \\
& Y_{r}=-K_{r} \beta_{r}=-K_{r}\left(\beta-\frac{l_{r}}{V} \gamma\right) \\
& N=d_{r}\left(X_{r r}-X_{r l}\right)
\end{aligned}
$$

Here, $K_{f}$ and $K_{r}$ are the equivalent cornering power of front and rear tire respectively. These values are calculated by using the full-vehicle model described in the section 5.1 to consider the effects of elasticity and friction of suspension and steering.

If driving force $F$ and DYC moment $N$ can be calculated by some control logic, then the target longitudinal forces of left and right rear wheel to be realized by TVD power unit become as follows from equation (5) and equation (10).

$$
\begin{aligned}
& X_{r r}=\frac{1}{2}\left(F+\frac{N}{d_{r}}\right) \\
& X_{r l}=\frac{1}{2}\left(F-\frac{N}{d_{r}}\right)
\end{aligned}
$$

\subsection{Longitudinal Driving Force Controller}

Let us suppose the desired value of vehicle speed, vehicle yaw rate and vehicle slip angle as $V_{r e f}, \gamma_{\text {ref }}$ and $\beta_{\text {ref }}$ respectively.

The desired vehicle driving force $F$ can be calculated as below by PI feedback control and equation (5).

$$
F=M \frac{d V_{r e f}}{d t}+K_{P F}\left(V_{r e f}-V\right)+K_{I F} \int\left(V_{r e f}-V\right) d t
$$

Here $K_{P F}$ is a proportional feedback gain and $K_{I F}$ is an integral feedback gain.

\subsection{Model Matching Controller of Lateral Dynamics}

\subsubsection{Dynamic Model of Vehicle Lateral Dynamics}

For the lateral dynamics, the state space form of the vehicle dynamics with TVD control becomes as follow from equation (6) and (7). 


$$
\begin{aligned}
\frac{d}{d t}\left[\begin{array}{l}
\beta \\
\gamma
\end{array}\right]= & {\left[\begin{array}{cc}
-\frac{2\left(K_{f}+K_{r}\right)}{M V} & -1-\frac{2\left(l_{f} K_{f}-l_{r} K_{r}\right)}{M V^{2}} \\
-\frac{2\left(l_{f} K_{f}-l_{r} K_{r}\right)}{I_{z}} & -\frac{2\left(l_{f}^{2} K_{f}+l_{r}^{2} K_{r}\right)}{I_{z} V}
\end{array}\right]\left[\begin{array}{l}
\beta \\
\gamma
\end{array}\right] } \\
& +\left[\begin{array}{c}
\frac{2 K_{f}}{M V} \\
\frac{2 l_{f} K_{f}}{I_{z}}
\end{array}\right] \frac{\delta_{s}}{G_{s}}+\left[\begin{array}{l}
0 \\
\frac{1}{I_{z}}
\end{array}\right] N
\end{aligned}
$$

Here, $\delta_{f}=\delta_{s} / \mathrm{G}_{s}\left(\delta_{s}\right.$ : steering wheel input angle, $G_{s}$ : steering gear ratio).

Now the matrix form of the state space system of equation (14) can be written as follows.

$$
\begin{aligned}
& \dot{x}=A x+B u+E \delta_{s} \\
& x=\left\lfloor\begin{array}{l}
\beta \\
\gamma
\end{array}\right\rfloor, \quad u=N \\
& A=\left[\begin{array}{cc}
-\frac{2\left(K_{f}+K_{r}\right)}{M V} & -1-\frac{2\left(l_{f} K_{f}-l_{r} K_{r}\right)}{M V^{2}} \\
-\frac{2\left(l_{f} K_{f}-l_{r} K_{r}\right)}{I_{z}} & -\frac{2\left(l_{f}^{2} K_{f}+l_{r}^{2} K_{r}\right)}{I_{z} V}
\end{array}\right] \\
& =\left[\begin{array}{ll}
a_{11} & a_{12} \\
a_{21} & a_{22}
\end{array}\right] \\
& B=\left\lfloor\begin{array}{c}
0 \\
1 \\
I_{z}
\end{array}\right\rfloor \\
& E=\left\lfloor\begin{array}{l}
\frac{2 K_{f}}{G_{s} M V} \\
\frac{2 l_{f} K_{f}}{G_{s} I_{z}}
\end{array}\right\rfloor
\end{aligned}
$$

Please note that the elements of the matrix A of the equation (15) as shown in the equation (16) are dependent on the vehicle velocity $V$, namely timevarying variables.

\subsubsection{Desired Dynamics Model for Lateral Motion}

The desired dynamics of vehicle yaw rate and vehicle slip angle are assumed as the first order lag function of steering wheel input as below.

$$
x_{d}=\left[\begin{array}{l}
\beta_{r e f} \\
\gamma_{r e f}
\end{array}\right]=\left\lfloor\begin{array}{l}
\frac{k_{\beta}}{1+s \tau_{\beta}} G_{\beta 0} \\
\frac{k_{\gamma}}{1+s \tau_{\gamma}} G_{\gamma 0}
\end{array}\right\rfloor \delta_{s}
$$

Here, $G_{\beta 0}$ and $G_{\gamma 0}$ are steady state gain of slip angle and yaw rate respectively from the steering input. $k_{\beta}$ and $k_{\gamma}$ are gain of desired slip angle and desired yaw rate from the steady state gain of each state variables. $\tau_{\beta}$ and $\tau_{\gamma}$ are time constant of desired slip angle and desired yaw rate as the first order lag function. Each state variables of slip angle and yaw rate at steady state can be calculated by solving the following equation

$$
0=A x_{0}+E \delta_{s}
$$

and be obtained as follow.

$$
\begin{aligned}
x_{0}= & -A^{-1} E \delta_{s} \\
= & -\frac{M I_{z} V^{2}}{4 K_{f} K_{r}\left(l_{f}+l_{r}\right)^{2}-2 M V^{2}\left(l_{f} K_{f}-l_{r} K_{r}\right)} \\
& \times\left[\begin{array}{c}
-\frac{4 K_{f} K_{r} l_{r}\left(l_{f}+l_{r}\right)}{M I_{z} V^{2}}+\frac{2 l_{f} K_{f}}{I_{z}} \\
\frac{-4 K_{f} K_{r}\left(l_{f}+l_{r}\right)}{M I_{z} V}
\end{array}\right] \frac{1}{G_{s}} \delta_{s}
\end{aligned}
$$

Thus, $G_{\beta 0}$ and $G_{\gamma 0}$ can be calculated as follows.

$$
\begin{aligned}
\left\lfloor\begin{array}{l}
G_{\beta 0} \\
G_{\gamma 0}
\end{array}\right] & =-\frac{M I_{z} V^{2}}{4 K_{f} K_{r}\left(l_{f}+l_{r}\right)^{2}-2 M V^{2}\left(l_{f} K_{f}-l_{r} K_{r}\right)} \\
& \times\left[\begin{array}{c}
-\frac{4 K_{f} K_{r} l_{r}\left(l_{f}+l_{r}\right)}{M I_{z} V^{2}}+\frac{2 l_{f} K_{f}}{I_{z}} \\
\frac{-4 K_{f} K_{r}\left(l_{f}+l_{r}\right)}{M I_{z} V}
\end{array}\right] \frac{1}{G_{s}}
\end{aligned}
$$

The state space form of the desired dynamics can be written as below from the equation (19).

$$
\dot{x}_{d}=A_{d} x_{d}+E_{d} \delta_{s}
$$

Here,

$$
\begin{aligned}
A_{d} & =\left\lfloor\begin{array}{cc}
-\frac{1}{\tau_{\beta}} & 0 \\
0 & -\frac{1}{\tau_{\gamma}}
\end{array}\right\rfloor \text { and } \\
E_{d} & =\left\lfloor\begin{array}{l}
\frac{k_{\beta}}{\tau_{\beta}} G_{\beta 0} \\
\frac{k_{\gamma}}{\tau_{\gamma}} G_{\gamma 0}
\end{array}\right\rfloor .
\end{aligned}
$$

\subsubsection{Model Matching Control of TVD}

A state equation of the error between desired values and actual values of state variables can be obtained as below by subtracting equation (23) from equation (15).

$$
\begin{aligned}
& \dot{e}=A e+B u+\left(A-A_{d}\right) x_{d}+\left(E-E_{d}\right) \delta_{s} \\
& e=x-x_{d}
\end{aligned}
$$

Let's assume the virtual control input $U$ as below.

$$
B U=B u+\left(A-A_{d}\right) x_{d}+\left(E-E_{d}\right) \delta_{s}
$$

Then the equation (24) can be transformed as below.

$$
\dot{e}=A e+B U
$$

Now we can design the feedback control gain $\mathrm{K}$ as

$$
U=-K e
$$

by using various linear control theories for the equation (26). Though, as mentioned above, the matrix $\mathrm{A}$ is 


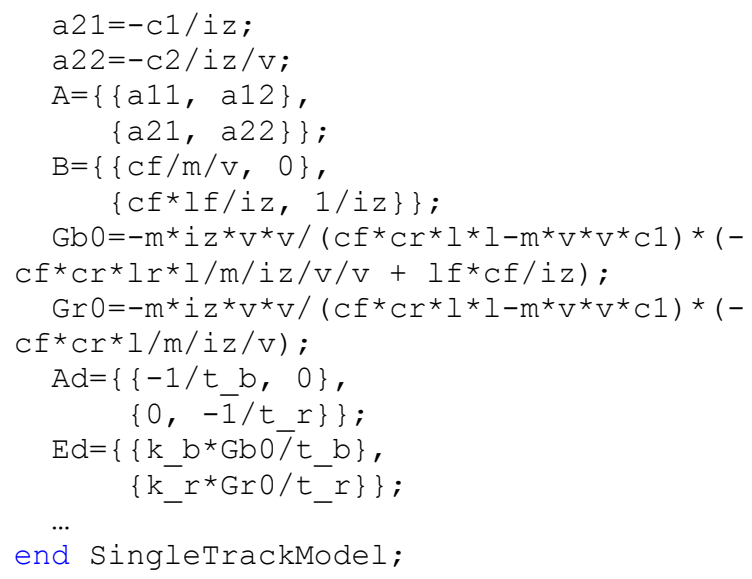

For comparison, the definition of the standard class of the state space system in MSL is as below.

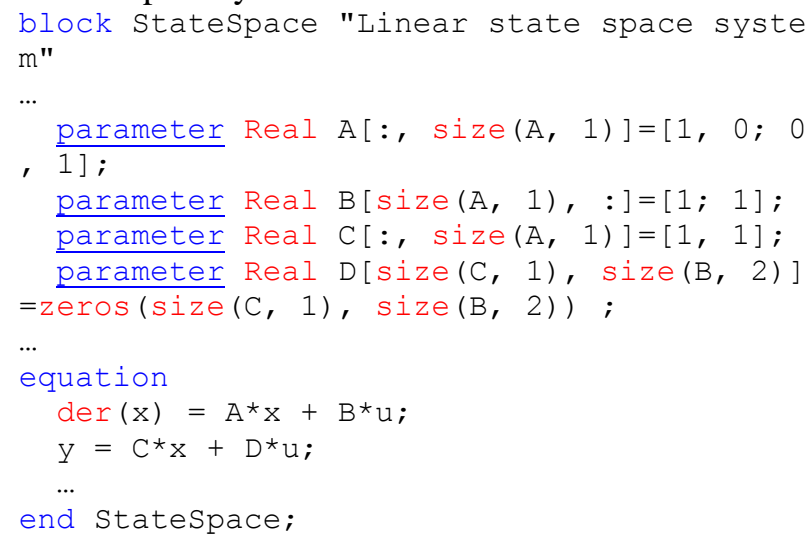

Also a new class of time-varying matrix gain to express the feedback gain by the equation (31) can be made by similar way.

Figure 3 shows a diagram of an example of a single track vehicle model combined with the desired vehicle dynamics model and the model matching controller.

Figure 4 shows a plot of vehicle speed and steering angle input used in the simulation by single track model. The vehicle accelerates from $10 \mathrm{~km} / \mathrm{h}$ to $100 \mathrm{~km} / \mathrm{h}$ between time $1 \mathrm{sec}$ to $10 \mathrm{sec}$. The steering angle moves as $1 \mathrm{~Hz}$ sinusoidal curve. For comparison, simple PI feedback of desired yaw rate and that of desired slip angle were also tested. The control law of both PI controllers became as follows respectively.

PI feedback of desired yaw rate:

$$
\left.N=K_{P \gamma}\left(\gamma_{\text {ref }}-\gamma\right)+K_{I \gamma}\right\rfloor\left(\gamma_{\text {ref }}-\gamma\right) d t
$$

PI feedback of desired slip angle:

$$
\left.N=K_{P \beta}\left(\beta_{r e f}-\beta\right)+K_{I \beta}\right\rfloor\left(\beta_{\text {ref }}-\beta\right) d t
$$

Desired dynamics was settled as $k_{\beta}=0.3, k_{\gamma}=1.0 . \tau_{\beta}$ and $\tau_{\gamma}$ are settled as corresponding value of cut-off frequency of $1.3 \mathrm{~Hz}$ as shown in the equation (19).

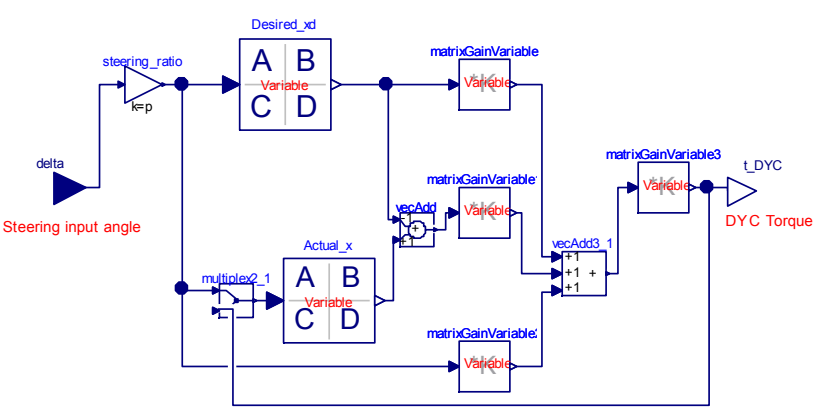

Figure 3. Modelica model of a single track model of vehicle and a controller
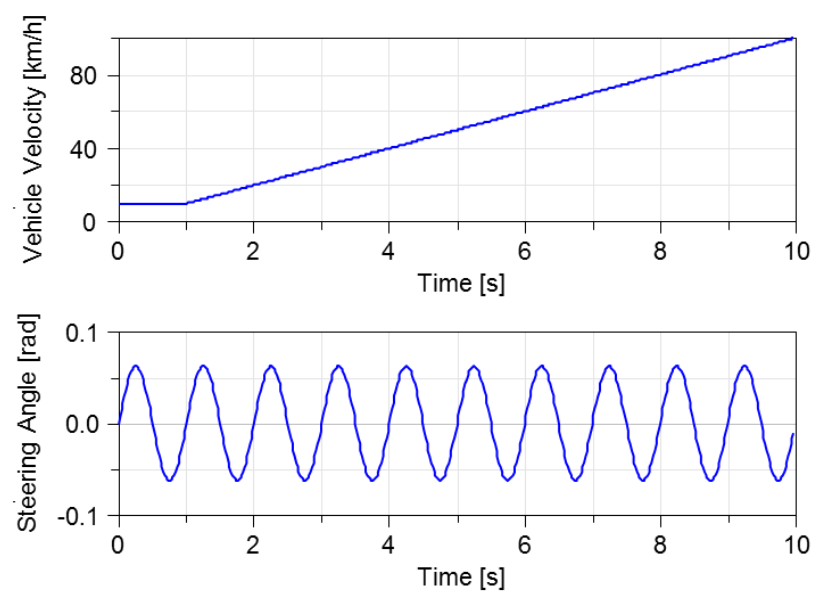

Figure 4. Plot of vehicle velocity and steering angle input

Figure 5 shows comparison of each control. The model matching control showed the best tracking performance of desired slip angle and desired yaw rate. Though, the control input $N$ was bigger than other controls and also the tracking error of yaw rate was bigger especially at the low vehicle speed. Also, it was impossible to let both of the vehicle slip angle and the yaw rate to exactly track the desired value simultaneously. This is because that there are two independent state variables while there is only one control input.

Robustness of the model matching control (MMC) was also checked. Figure 6 shows comparison of the simulation results of single track model when there are perturbation for the vehicle mass $\mathrm{M}$ and tire cornering power CP. For comparison, the result of yaw rate feedback control is also overlaid. MMC showed a good robustness against such parameter perturbations.

It is of course necessary to check the robustness of the control when parameter error of the plant and also other additional effects such as non-linearity and losses exist in the actual world. To do this, simulation tests using full vehicle model was also done as mentioned in the following section. 

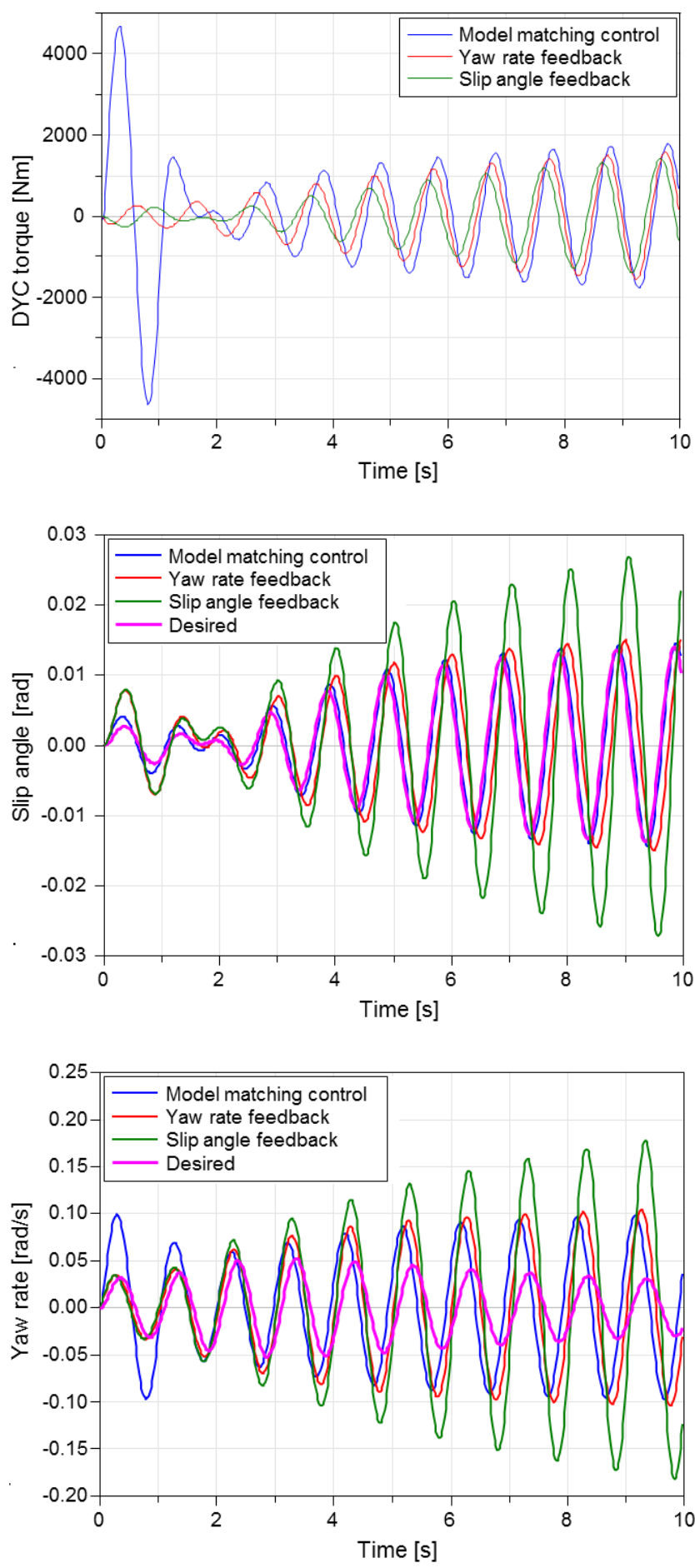

Figure 5. Simulation results by single track model

\section{Simulation by Full Vehicle Model}

\subsection{Construction of the Full-Vehicle Model}

The similar full vehicle model as previous research (Hirano, 2015) was used for full-vehicle simulation. The model was developed based on Vehicle Dynamics Library (Modelon, 2014) and was built as a full 3 dimensional (3D) multi-body-dynamic system (MBS) model. Component models of control systems such as TVD gearbox, electric motor and inverter were added with the full vehicle model. Figure 7 shows the top level of the model hierarchy of the full vehicle test model and also the power train model with the controller.
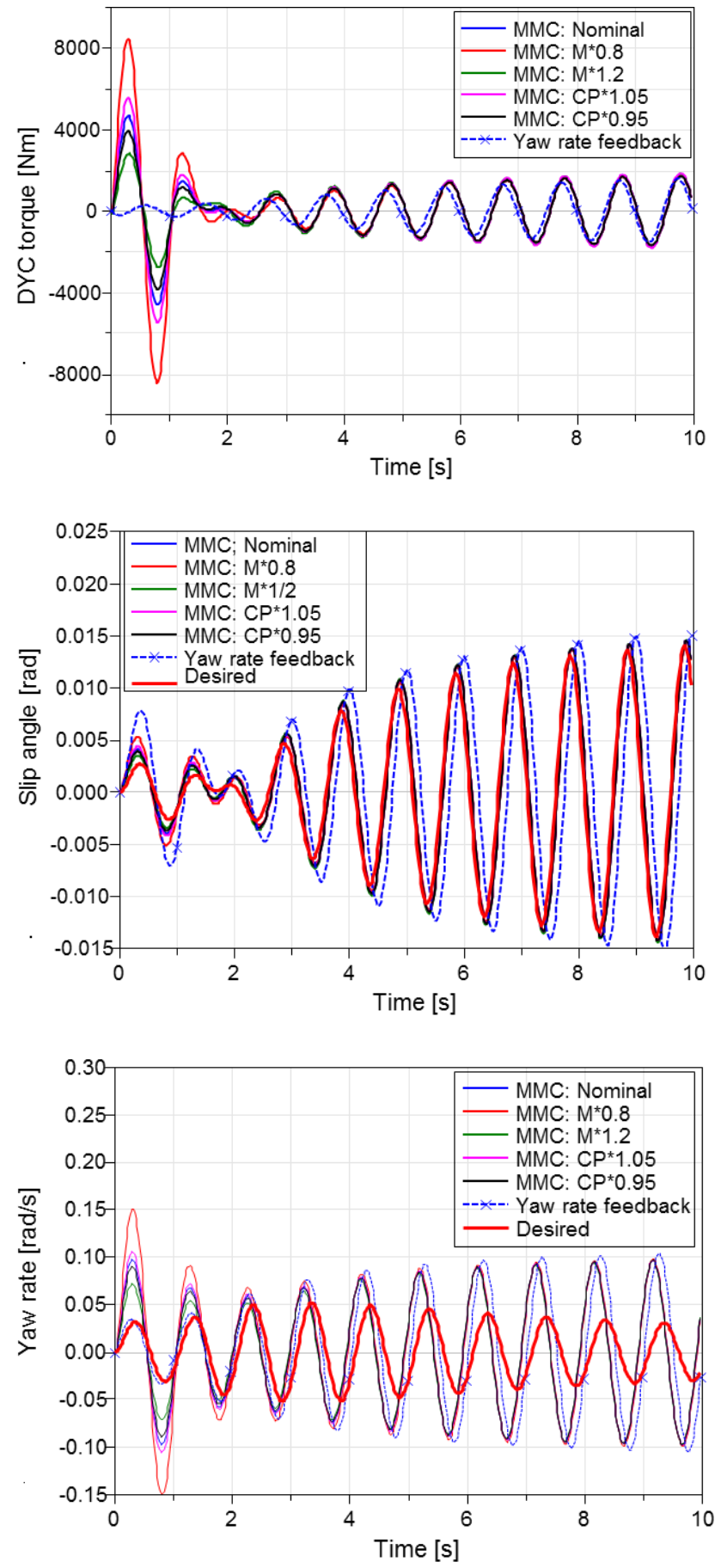

Figure 6. Robustness check by single track model 


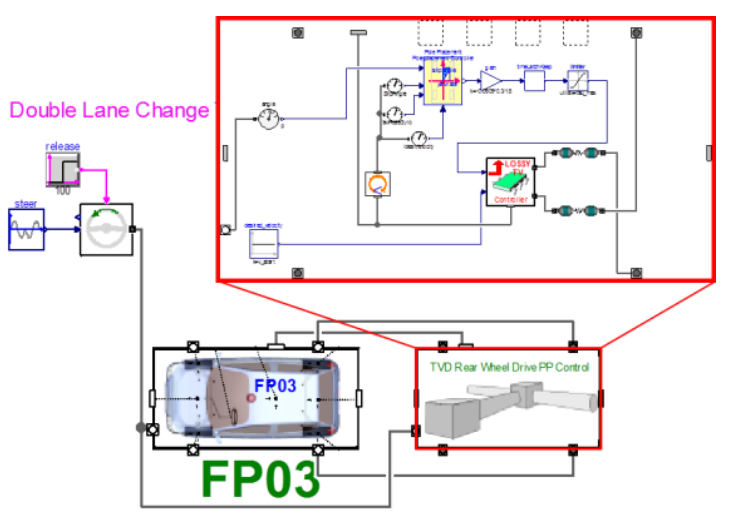

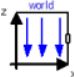

Figure 7. Structure of full vehicle test model

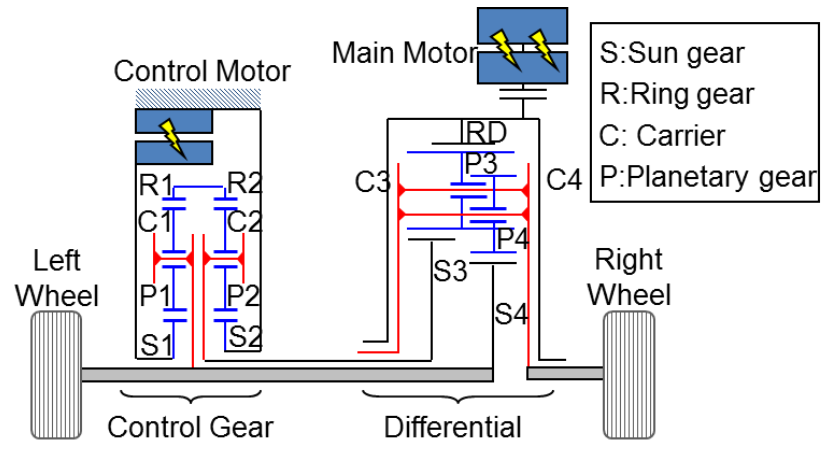

Figure 8. Torque vectoring differential (TVD) driveline

For the TVD gear train, a driveline structure referencing the MUTE project of the Technische Universität München (TUM) (Höhn et al., 2013) was selected. The TVD model was constructed using Power Train Library (DLR, 2013). Figure 8 shows the configuration of the gear trains. Torque from the main motor is distributed equally to the left wheel and the right wheel through the differential gear. The torque distribution between the left wheel and the right wheel can be controlled by changing the torque input of the control motor.

3D MBS model of suspension, steering and body were installed to calculate vehicle dynamics characteristics. Suspension model was constructed as an assembled model of each suspension linkage, joints and force elements such as spring, damper and bushing. Non-linear tire model based on 'Magic Formula' model (Pacejka02) was used to calculate combined lateral force and longitudinal force of each tire. Steering model considered the characteristics of viscous friction of steering gear box and steering shaft as well as steering shaft stiffness. By these detailed models, it became possible to analyze the effects of steering angle change and camber angle change caused by vehicle roll, side force and tire aligning torque.

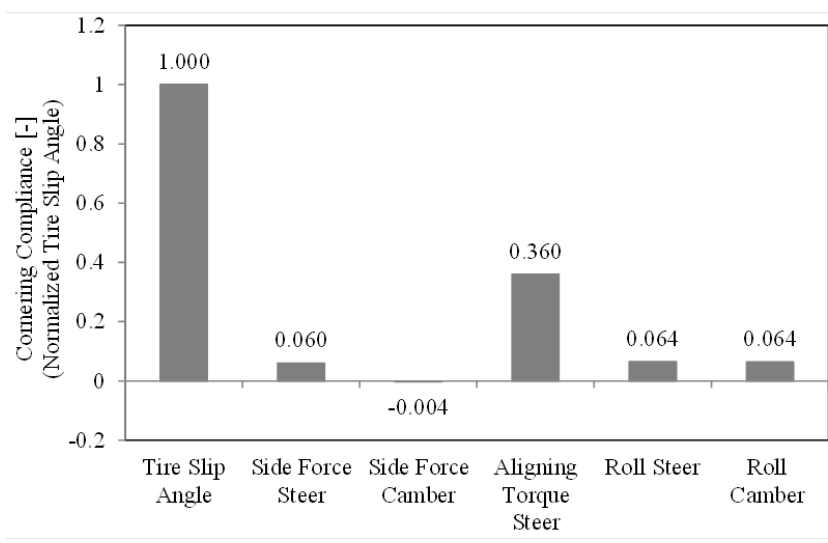

Figure 9. Effect of suspension characteristics to cornering compliance coefficient. (Normalized by the effect of tire slip angle.)

Figure 9 shows an analysis result about the effect of suspension characteristics to cornering compliance coefficient for an example of front double wish-born suspension. The coefficients are normalized by the effect of tire slip angle change. The equivalent cornering power coefficients were calculated by following equation.

$$
\varepsilon_{f, r}=\frac{\frac{1}{C_{f, r}}}{\frac{1}{C_{f, r}}+\left\{W_{f, r}\left(\frac{\partial \delta}{\partial F}\right)_{f, r}+W_{f, r}\left(\frac{\partial \gamma}{\partial F}\right)_{f, r} \frac{C_{s_{f, r}}}{C_{f, r}}+W_{f, r} \zeta_{f, r}\left(\frac{\partial \delta}{\partial M}\right)_{f, r}+\phi\left(\frac{\partial \delta}{\partial \phi}\right)_{f, r}+\phi\left(\frac{\partial \gamma}{\partial \phi}\right)_{f, r} \frac{C_{s_{f, r}}}{C_{f, r}}\right\}}
$$

Here, $C_{f}$ and $C_{r}$ are the cornering power of tire itself. The terms in the curly brace of the denominator of the above equation indicates each effect shown in Figure 9 respectively. Those are the effects by side force steer, side force camber, aligning torque steer, roll steer and roll camber respectively. Finally, the equivalent cornering power coefficients of front tires and rear tires were calculated as $\varepsilon_{f}$ and $\varepsilon_{r}$ respectively. These values are used to calculate the equivalent cornering power of each wheel shown in the equation (8) and equation (9) as bellows.

$$
\begin{aligned}
& K_{f}=\varepsilon_{f} C_{f} \\
& K_{r}=\varepsilon_{r} C_{r}
\end{aligned}
$$




\subsection{Results of Full Vehicle Simulation}

Figure 10 shows the results of a double lane change test by the full vehicle model. Steering angle was given as a series of sinusoidal curves at a constant vehicle velocity of $100[\mathrm{~km} / \mathrm{h}]$. The model matching control showed better performance of tracking desired slip angle than the yaw rate feedback control. On the other hand, the yaw rate feedback control showed better performance for tracking the desired yaw rate, though this result can be expected naturally. Additionally, it became clear that the result of the vehicle motion by the model matching control was smoother than that by the PI yaw rate feedback control of desired yaw rate. The reason of this is assumed that feedforward part of model matching control works to improve the response. On the other hand, PI feedback control of the desired slip angle became unstable.

Figure 11 shows the result of full vehicle model simulation for the side wind test. Here, side wind of $20[\mathrm{~m} / \mathrm{s}$ ] blows while Time $=2$ [s] to 3.5 [s]. The vehicle runs at $120[\mathrm{~km} / \mathrm{h}]$ and the steering wheel angle is kept to zero. Here, the similar result as the side wind test was obtained. The model matching control was good at tracking performance of the desired slip angle, and the PI feedback control of the desired yaw rate was good at tracking performance of the desired yaw rate. Also it is indicated that the control ability against steady deviation for the model matching controller is not enough. This indicates the necessity of modifying the model matching controller to introduce first order servo control by considering the integral of the error. Anyway both controls showed good performance of vehicle stabilization against the side wind than when no control was applied.

\section{Conclusions}

Model matching control of TVD was researched by using both linear single track model of vehicle dynamics and multi-physics large-scale full vehicle model. The following conclusions were obtained.

(i) Proposed model matching control showed a good performance especially for the tracking of the desired slip angle.

(ii) On the other hand, simple PI feedback control of desired yaw rate was good at tracking the desired yaw rate than the model matching control.

(iii) Improving the model matching controller to realize servo control of steady error deviation is necessary for future work.

Also for future work, the effect of drive shaft stiffness for TVD control should be investigated. More sophisticated control of tire slip and drive train oscillation should be researched also satisfying the requirement for the vehicle dynamics performance.

\section{References}

DLR, PowerTrain Library Users Guide (Version 2.1.0), 2013

Y. Hirano, S. Inoue and J. Ota, Model-based Development of Future Small EVs using Modelica, Proceedings of Modelica Conference 2014, 2014.

Y. Hirano, S. Inoue and J. Ota, Model Based Development of Future Small Electric Vehicle by Modelica, Proceedings of Modelica Conference 2015, 2015.

B. Höhn et al., Torque Vectoring Driveline for Electric Vehicle, Proceedings of the FISITA 2012 World Automotive Congress, Vol. 191, pp. 585-593, 2013.

Modelon, A.B., Vehicle Dynamics library Users Guide (Version 1.8), 2014 

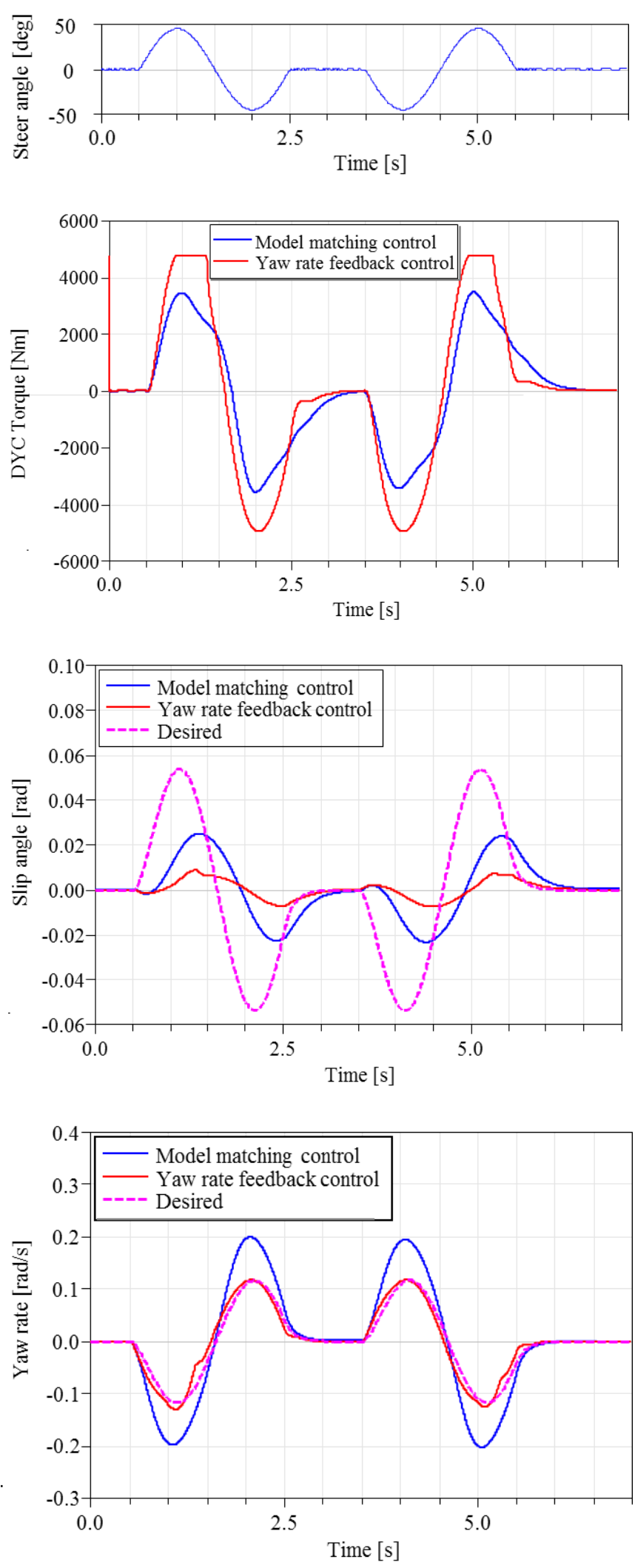

Figure 10. Simulation result of double lane change test by full vehicle model
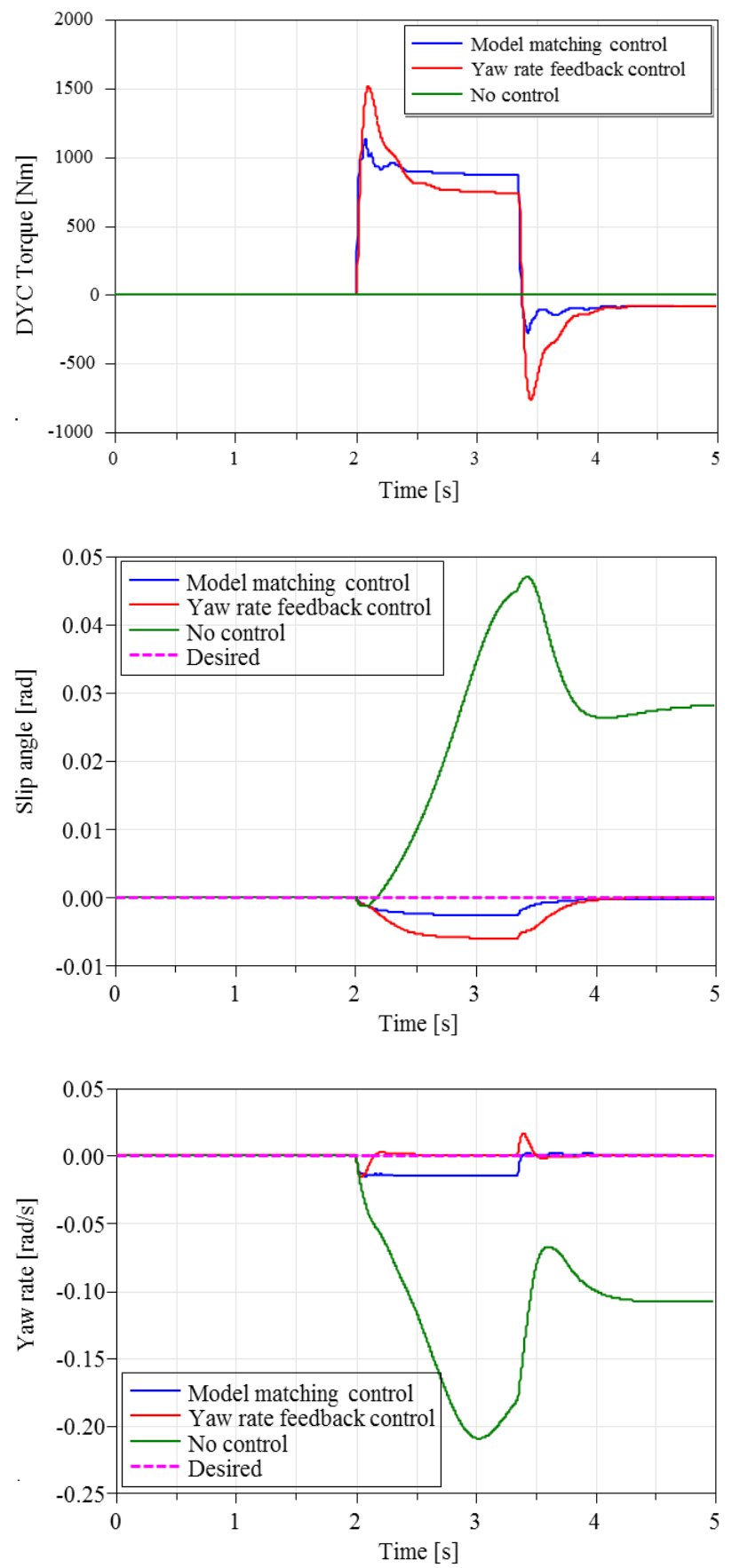

Figure 11. Simulation result of side wind test by full vehicle model 